Supplement of The Cryosphere, 12, 3045-3065, 2018

https://doi.org/10.5194/tc-12-3045-2018-supplement

(C) Author(s) 2018. This work is distributed under

the Creative Commons Attribution 4.0 License.

(c) (1)

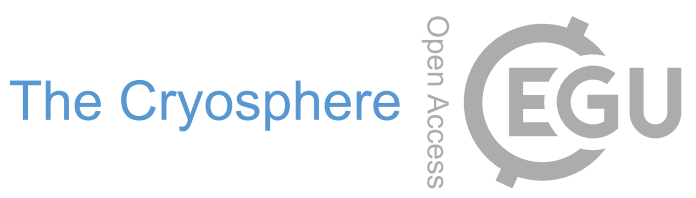

Supplement of

\title{
Dual-satellite (Sentinel-2 and Landsat 8) remote sensing of supraglacial lakes in Greenland
}

Andrew G. Williamson et al.

Correspondence to: Andrew G. Williamson (agw41@alumni.cam.ac.uk)

The copyright of individual parts of the supplement might differ from the CC BY 4.0 License. 
We followed the methods of Pope et al. (2016; their Supplement) to calculate the coefficient for the losses in upward and downward travel through the water column $\left(g ; \mathrm{m}^{-1}\right)$ for the red and green bands of Sentinel-2. The relationship from Smith and Baker (1981; their Eq. (5)) states that:

$K_{d}=a+\frac{1}{2} b$,

where $K_{d}$ is the diffuse attenuation coefficient for the clearest natural freshwaters, $a$ is the absorption coefficient for pure water, and $b$ is the backscattering coefficient for molecular (Rayleigh) scattering in freshwater.

We then used the relationship from Sneed and Hamilton (2007; their Eq. (3)), which indicates that:

$g \approx K_{d}+a D_{u}$

where $D_{u}$ is an upwelling light distribution or the reciprocal of the upwelling average cosine (Mobley, 1994). Since $K_{d} \approx a D_{u}$ (Maritorena et al., 1994), $g$ can simply be calculated (using measured values of $a$ and $b$ ) with the following:

$g=2\left(K_{d}\right)$,

meaning that Eq. (S1) could then be used to derive the following relationship:

$g=2\left(a+\frac{1}{2} b\right)$

For $a$, we followed Pope et al. (2016) and took the value from Pope and Fry (1997; their Table 3) to replace that from Smith and Baker (1981; their Table 1), and, for b, we took the value from Buiteveld et al. (1994; their Table 1) for each wavelength of Sentinel-2's red and green bands. This allowed a preliminary $g$ value to be calculated for each of Sentinel-2's red and green bands by using Eq. (S4) for each wavelength of the red band (from 646-684 nm in $1 \mathrm{~nm}$ increments) and green band (from 537-582 nm in $1 \mathrm{~nm}$ increments). To derive final $g$ values for each band, following Pope et al. (2016), Sentinel-2's spectral response function (ESA, 2017) was used, akin to the data available for Landsat 8 (Barsi et al., 2014), and average weighted values were calculated according to the spectral response for each wavelength of the red and green bands.

This produced final $g$ values for Sentinel-2's red band of 0.8304 and for Sentinel-2's green band of 0.1413, which compared with the lower $g$ values for Landsat 8's red band of 0.7507 and for Landsat 8's green band of 0.1279 (Pope et al., 2016). 


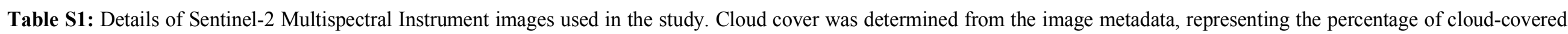

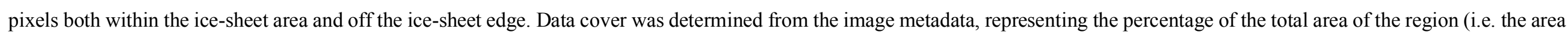
on the ice sheet plus the ice-marginal area) for which satellite data were collected.

\begin{tabular}{|c|c|c|c|c|c|c|c|c|}
\hline Scene ID & $\begin{array}{l}\text { UTM } \\
\text { zone }\end{array}$ & $\begin{array}{c}\text { Latitude } \\
\text { band }\end{array}$ & Square & Date & Year & Sequence & $\begin{array}{c}\text { Cloud } \\
\text { cover }(\%)\end{array}$ & $\begin{array}{c}\text { Data } \\
\text { cover }(\%)\end{array}$ \\
\hline S2A_OPER_MSI_L1C_DS_MTI_20160502T202602_S20160502T151916_N02.01 & $22 \mathrm{~N}$ & $\mathrm{~W}$ & EC & 02 May & 2016 & 0 & 38.9 & 100.0 \\
\hline S2A_OPER_MSI_L1C_DS_SGS_20160505T205541_S20160505T152915_N02.02 & $22 \mathrm{~N}$ & $\mathrm{~W}$ & $\mathrm{EC}$ & 05 May & 2016 & 0 & 44.7 & 100.0 \\
\hline S2A_OPER_MSI_L1C_DS_MTI_20160515T203516_S20160515T152912_N02.02 & $22 \mathrm{~N}$ & $\mathrm{~W}$ & $\mathrm{EC}$ & 15 May & 2016 & 0 & 5.4 & 100.0 \\
\hline S2A_OPER_MSI_L1C_DS_SGS_20160518T210306_S20160518T154153_N02.02 & $22 \mathrm{~N}$ & $\mathrm{~W}$ & $\mathrm{EC}$ & 18 May & 2016 & 0 & 31.4 & 38.3 \\
\hline S2A_OPER_MSI_L1C_DS_MTI_20160522T202416_S20160522T151915_N02.02 & $22 \mathrm{~N}$ & $\mathrm{~W}$ & $\mathrm{EC}$ & 22 May & 2016 & 0 & 29.8 & 100.0 \\
\hline S2A_OPER_MSI_L1C_DS_MTI_20160529T201543_S20160529T150918_N02.02 & $22 \mathrm{~N}$ & $\mathrm{~W}$ & $\mathrm{EC}$ & 29 May & 2016 & 0 & 66.1 & 94.3 \\
\hline S2A_OPER_MSI_L1C_DS_SGS_20160601T173946_S20160601T151916_N02.02 & $22 \mathrm{~N}$ & $\mathrm{~W}$ & $\mathrm{EC}$ & 01 Jun & 2016 & 0 & 18.1 & 99.9 \\
\hline S2A_OPER_MSI_L1C_DS_MTI_20160604T203539_S20160604T152915_N02.02 & $22 \mathrm{~N}$ & $\mathrm{~W}$ & $\mathrm{EC}$ & 04 Jun & 2016 & 0 & 60.0 & 99.9 \\
\hline S2A_OPER_MSI_L1C_DS_SGS_20160608T202646_S20160608T150916_N02.02 & $22 \mathrm{~N}$ & $\mathrm{~W}$ & $\mathrm{EC}$ & 08 Jun & 2016 & 0 & 16.2 & 94.2 \\
\hline S2A_OPER_MSI_L1C_DS_MTI_20160611T202435_S20160611T151937_N02.02 & $22 \mathrm{~N}$ & $\mathrm{~W}$ & $\mathrm{EC}$ & $11 \mathrm{Jun}$ & 2016 & 0 & 42.5 & 100.0 \\
\hline S2A_OPER_MSI_L1C_DS_SGS_20160617T192017_S20160617T153910_N02.04 & $22 \mathrm{~N}$ & $\mathrm{~W}$ & $\mathrm{EC}$ & 17 Jun & 2016 & 0 & 25.7 & 38.9 \\
\hline S2A_OPER_MSI_L1C_DS_SGS_20160618T202441_S20160618T151305_N02.04 & $22 \mathrm{~N}$ & $\mathrm{~W}$ & $\mathrm{EC}$ & 18 Jun & 2016 & 0 & 46.7 & 93.2 \\
\hline S2A_OPER_MSI_L1C_DS_MTI_20160621T220258_S20160621T151912_N02.04 & $22 \mathrm{~N}$ & $\mathrm{~W}$ & $\mathrm{EC}$ & 21 Jun & 2016 & 0 & 4.3 & 100.0 \\
\hline S2A_OPER_MSI_L1C_DS_MTI_20160624T203434_S20160624T152911_N02.04 & $22 \mathrm{~N}$ & $\mathrm{~W}$ & $\mathrm{EC}$ & 24 Jun & 2016 & 0 & 16.0 & 100.0 \\
\hline S2A_OPER_MSI_L1C_DS_SGS_20160628T202858_S20160628T150914_N02.04 & $22 \mathrm{~N}$ & $\mathrm{~W}$ & $\mathrm{EC}$ & 28 Jun & 2016 & 0 & 0.1 & 94.0 \\
\hline S2A_OPER_MSI_L1C_DS_MTI_20160701T202434_S20160701T151913_N02.04 & $22 \mathrm{~N}$ & $\mathrm{~W}$ & $\mathrm{EC}$ & $01 \mathrm{Jul}$ & 2016 & 0 & 73.3 & 100.0 \\
\hline S2A_OPER_MSI_L1C_DS_SGS_20160707T192822_S20160707T153908_N02.04 & $22 \mathrm{~N}$ & $\mathrm{~W}$ & $\mathrm{EC}$ & $07 \mathrm{Jul}$ & 2016 & 0 & 0.0 & 38.6 \\
\hline S2A_OPER_MSI_L1C_DS_SGS_20160708T202909_S20160708T151305_N02.04 & $22 \mathrm{~N}$ & $\mathrm{~W}$ & $\mathrm{EC}$ & $08 \mathrm{Jul}$ & 2016 & 1 & 1.6 & 94.2 \\
\hline S2A_OPER_MSI_L1C_DS_SGS_20160711T203749_S20160711T151912_N02.04 & $22 \mathrm{~N}$ & $\mathrm{~W}$ & EC & $11 \mathrm{Jul}$ & 2016 & 0 & 0.1 & 100.0 \\
\hline S2A_OPER_MSI_L1C_DS_MTI_20160714T203553_S20160714T152910_N02.04 & $22 \mathrm{~N}$ & $\mathrm{~W}$ & $\mathrm{EC}$ & $14 \mathrm{Jul}$ & 2016 & 0 & 0.3 & 99.9 \\
\hline S2A_OPER_MSI_L1C_DS_SGS_20160718T202939_S20160718T150915_N02.04 & $22 \mathrm{~N}$ & W & $\mathrm{EC}$ & $18 \mathrm{Jul}$ & 2016 & 0 & 23.7 & 94.2 \\
\hline
\end{tabular}




\begin{tabular}{|c|c|c|c|c|c|c|c|c|}
\hline Scene ID & $\begin{array}{l}\text { UTM } \\
\text { zone }\end{array}$ & $\begin{array}{c}\text { Latitude } \\
\text { band }\end{array}$ & Square & Date & Year & Sequence & $\begin{array}{c}\text { Cloud } \\
\operatorname{cover}(\%)\end{array}$ & $\begin{array}{c}\text { Data } \\
\operatorname{cover}(\%)\end{array}$ \\
\hline S2A_OPER_MSI_L1C_DS_MTI_20160721T202530_S20160721T151913_N02.04 & $22 \mathrm{~N}$ & W & EC & $21 \mathrm{Jul}$ & 2016 & 0 & 0.0 & 100.0 \\
\hline S2A_OPER_MSI_L1C_DS_SGS_20160724T205100_S20160724T153051_N02.04 & $22 \mathrm{~N}$ & $\mathrm{~W}$ & $\mathrm{EC}$ & $24 \mathrm{Jul}$ & 2016 & 0 & 0.0 & 99.9 \\
\hline S2A_OPER_MSI_L1C_DS_SGS_20160727T192945_S20160727T153910_N02.04 & $22 \mathrm{~N}$ & $\mathrm{~W}$ & $\mathrm{EC}$ & $27 \mathrm{Jul}$ & 2016 & 0 & 0.0 & 38.2 \\
\hline S2A_OPER_MSI_L1C_DS_SGS_20160728T202436_S20160728T151306_N02.04 & $22 \mathrm{~N}$ & W & $\mathrm{EC}$ & $28 \mathrm{Jul}$ & 2016 & 0 & 63.6 & 94.3 \\
\hline S2A_OPER_MSI_L1C_DS_SGS_20160731T203724_S20160731T151913_N02.04 & $22 \mathrm{~N}$ & W & $\mathrm{EC}$ & $31 \mathrm{Jul}$ & 2016 & 0 & 0.0 & 100.0 \\
\hline S2A_OPER_MSI_L1C_DS_MTI_20160803T203554_S20160803T152910_N02.04 & $22 \mathrm{~N}$ & $\mathrm{~W}$ & $\mathrm{EC}$ & 03 Aug & 2016 & 0 & 3.1 & 100.0 \\
\hline S2A_OPER_MSI_L1C_DS_MTI_20160806T204409_S20160806T153909_N02.04 & $22 \mathrm{~N}$ & $\mathrm{~W}$ & $\mathrm{EC}$ & 06 Aug & 2016 & 0 & 14.1 & 38.1 \\
\hline S2A_OPER_MSI_L1C_DS_SGS_20160807T202758_S20160807T150914_N02.04 & $22 \mathrm{~N}$ & $\mathrm{~W}$ & $\mathrm{EC}$ & 07 Aug & 2016 & 0 & 55.9 & 94.4 \\
\hline S2A_OPER_MSI_L1C_DS_MTI_20160810T202535_S20160810T151912_N02.04 & $22 \mathrm{~N}$ & $\mathrm{~W}$ & $\mathrm{EC}$ & 10 Aug & 2016 & 0 & 13.0 & 100.0 \\
\hline S2A_OPER_MSI_L1C_DS_SGS_20160813T204833_S20160813T153046_N02.04 & $22 \mathrm{~N}$ & W & $\mathrm{EC}$ & 13 Aug & 2016 & 0 & 0.0 & 100.0 \\
\hline S2A_OPER_MSI_L1C_DS_SGS_20160817T202447_S20160817T151257_N02.04 & $22 \mathrm{~N}$ & W & $\mathrm{EC}$ & 17 Aug & 2016 & 0 & 0.7 & 94.4 \\
\hline S2A_OPER_MSI_L1C_DS_MTI_20160830T202522_S20160830T151909_N02.04 & $22 \mathrm{~N}$ & $\mathrm{~W}$ & $\mathrm{EC}$ & 30 Aug & 2016 & 0 & 2.2 & 100.0 \\
\hline S2A_OPER_MSI_L1C_DS_SGS_20160902T204312_S20160902T153051_N02.04 & $22 \mathrm{~N}$ & W & EC & 02 Sep & 2016 & 0 & 0.7 & 100.0 \\
\hline S2A_OPER_MSI_L1C_DS_SGS_20160906T202733_S20160906T151257_N02.04 & $22 \mathrm{~N}$ & W & $\mathrm{EC}$ & 06 Sep & 2016 & 0 & 35.9 & 94.5 \\
\hline S2A_OPER_MSI_L1C_DS_SGS_20160909T203815_S20160909T151907_N02.04 & $22 \mathrm{~N}$ & W & EC & 09 Sep & 2016 & 0 & 21.0 & 100.0 \\
\hline S2A_OPER_MSI_L1C_DS_MTI_20160912T203709_S20160912T152817_N02.04 & $22 \mathrm{~N}$ & W & $\mathrm{EC}$ & 12 Sep & 2016 & 0 & 0.0 & 100.0 \\
\hline S2A_OPER_MSI_L1C_DS_SGS_20160915T210159_S20160915T154130_N02.04 & $22 \mathrm{~N}$ & W & $\mathrm{EC}$ & 15 Sep & 2016 & 0 & 1.7 & 38.2 \\
\hline S2A_OPER_MSI_L1C_DS_MTI_20160926T201345_S20160926T150955_N02.04 & $22 \mathrm{~N}$ & $\mathrm{~W}$ & $\mathrm{EC}$ & $26 \mathrm{Sep}$ & 2016 & 0 & 0.3 & 93.9 \\
\hline
\end{tabular}


Table S2: Details of Landsat 8 Operational Land Imager images used in the study. Cloud cover was calculated as the percentage of pixels both on and off the ice sheet that were obscured by clouds (i.e. those with a band- 6 or SWIR value $>0.100$; Sect. 2.2.1). Data cover was calculated as a percentage of the total area of the region (i.e. the area on the ice sheet plus the ice-marginal area) for which satellite data had been collected. The asterisk denotes that the image was used for comparing lake areas calculated from the two satellites and for validating the lake depths from Sentinel-2, but that the image was not used for lake tracking because a contemporaneous higher-resolution, and therefore more favourable, Sentinel-2 image was available.

\begin{tabular}{ccccccc}
\hline Scene ID & Path & Row & Date & Year & Cloud cover (\%) & Data cover (\%) \\
\hline LC80100112016149LGN01 & 010 & 011 & 28 May & 2016 & 85.9 & 95.1 \\
LC80080112016151LGN01 & 008 & 011 & 30 May & 2016 & 8.4 & 72.8 \\
LC80822332016157LGN01 & 082 & 233 & 05 Jun & 2016 & 17.3 & 100.0 \\
LC80090112016158LGN01 & 009 & 011 & 06 Jun & 2016 & 21.6 & 100.0 \\
LC80100112016165LGN01 & 010 & 011 & 13 Jun & 2016 & 43.9 & 95.3 \\
LC80090112016174LGN01 & 009 & 011 & 22 Jun & 2016 & 33.9 & 100.0 \\
LC80100112016181LGN01 & 010 & 011 & 29 Jun & 2016 & 35.0 & 95.2 \\
LC80080112016183LGN01 & 008 & 011 & 01 Jul* & 2016 & 60.8 & 72.6 \\
LC80100112016197LGN01 & 010 & 011 & 15 Jul & 2016 & 21.0 & 95.1 \\
LC80812332016198LGN01 & 081 & 233 & 16 Jul & 2016 & 19.0 & 93.7 \\
LC80080112016199LGN01 & 008 & 011 & 17 Jul & 2016 & 5.9 & 72.8 \\
LC80100112016213LGN01 & 010 & 011 & 31 Jul & 2016 & 18.5 & 95.2 \\
LC80080112016215LGN01 & 008 & 011 & 02 Aug & 2016 & 12.2 & 72.6 \\
LC80090112016222LGN01 & 009 & 011 & 09 Aug & 2016 & 18.4 & 100.0 \\
LC80100112016229LGN01 & 010 & 011 & 16 Aug & 2016 & 18.3 & 95.2 \\
LC80080112016231LGN01 & 008 & 011 & 18 Aug & 2016 & 6.5 & 72.7 \\
LC80080112016247LGN01 & 008 & 011 & 03 Sep & 2016 & 8.8 & 73.2 \\
\hline
\end{tabular}




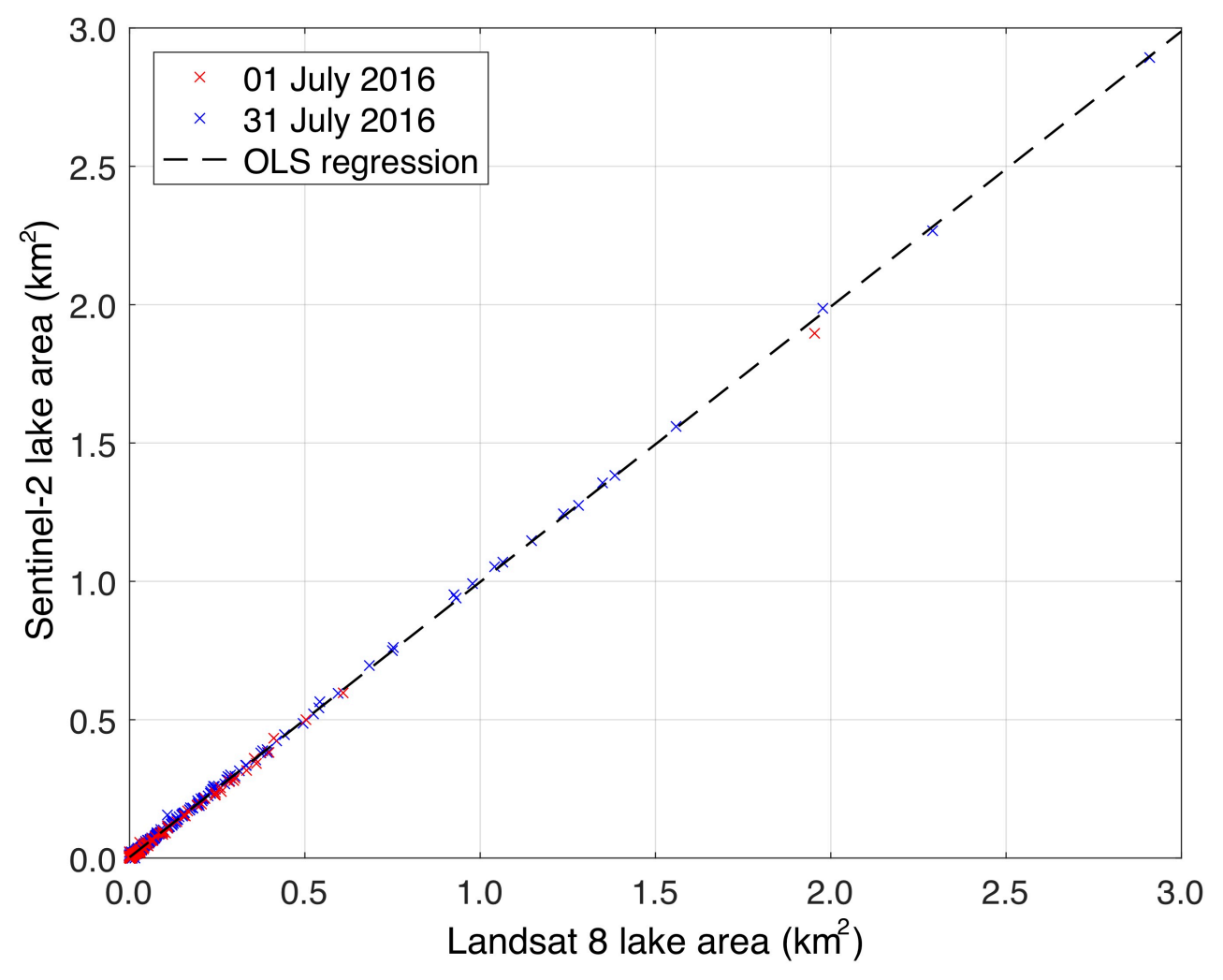

Figure S1: The very strong relationship between Landsat 8 and Sentinel-2 lake areas for the days of overlapping imagery (1 July and 31 July) in 2016. The 594 lake areas used for comparisons were derived using the Normalised Difference Water Index approach with threshold values of 0.25 for both types of imagery. The black dashed line shows an ordinary least-squares (OLS) linear regression, which can explain $99.9 \%\left(\mathrm{R}^{2}=0.999 ; p=0.000\right)$ of the variance in the data. The root-mean square error of $0.007 \mathrm{~km}^{2}$ (i.e. seven Sentinel-2 pixels) between the two sets of lake areas is therefore remarkably small. 


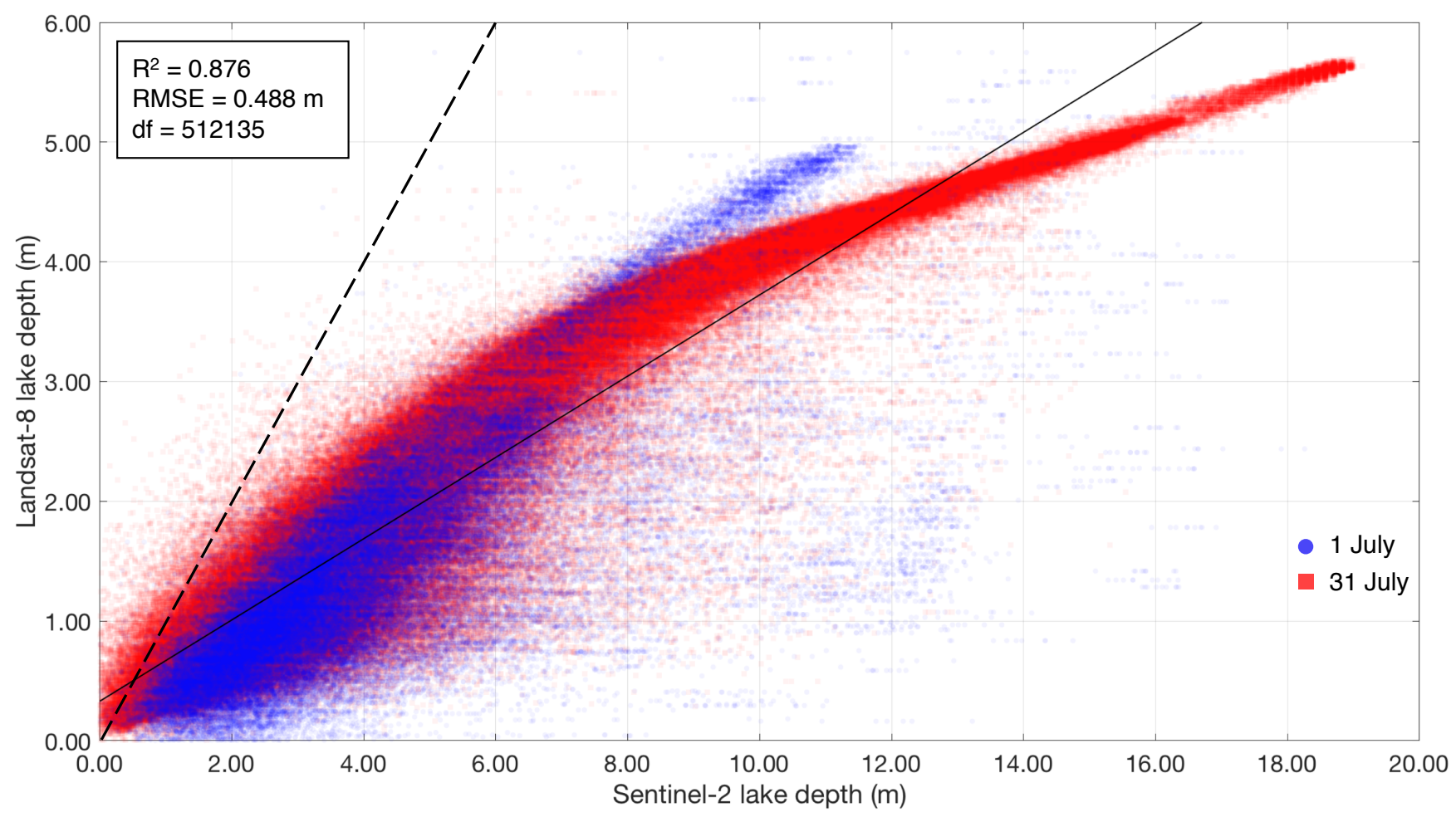

Figure S2: Comparison of lake depths calculated using the physically based method for Sentinel-2 (with the green band) and for Landsat 8 (with the average depths from the red and panchromatic bands). Degrees of freedom ("df" in this figure) $=512,135$. The solid black line shows an OLS linear regression and the dashed black line shows a 1:1 relation. The $\mathrm{R}^{2}$ value indicates that the regression explains $87.6 \%$ of the variance in the data. The RMSE of $0.488 \mathrm{~m}$ shows the error associated with calculating the Sentinel-2 lake depths using this relationship.

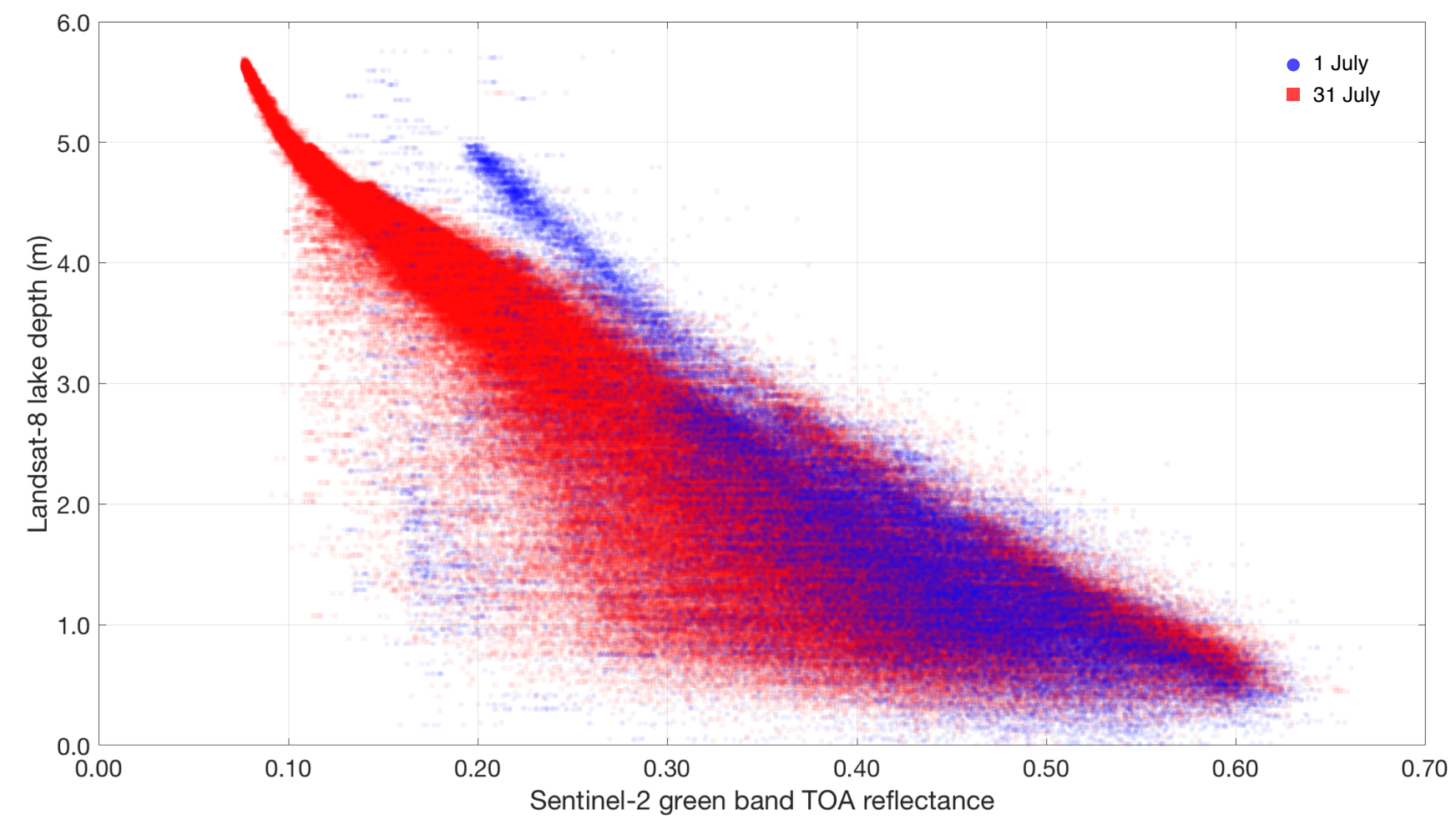

Figure S3: Scatter plot to show the relationship between Sentinel-2 green band top-of-atmosphere (TOA) reflectance and Landsat 8 lake depth for 430,650 data points. 


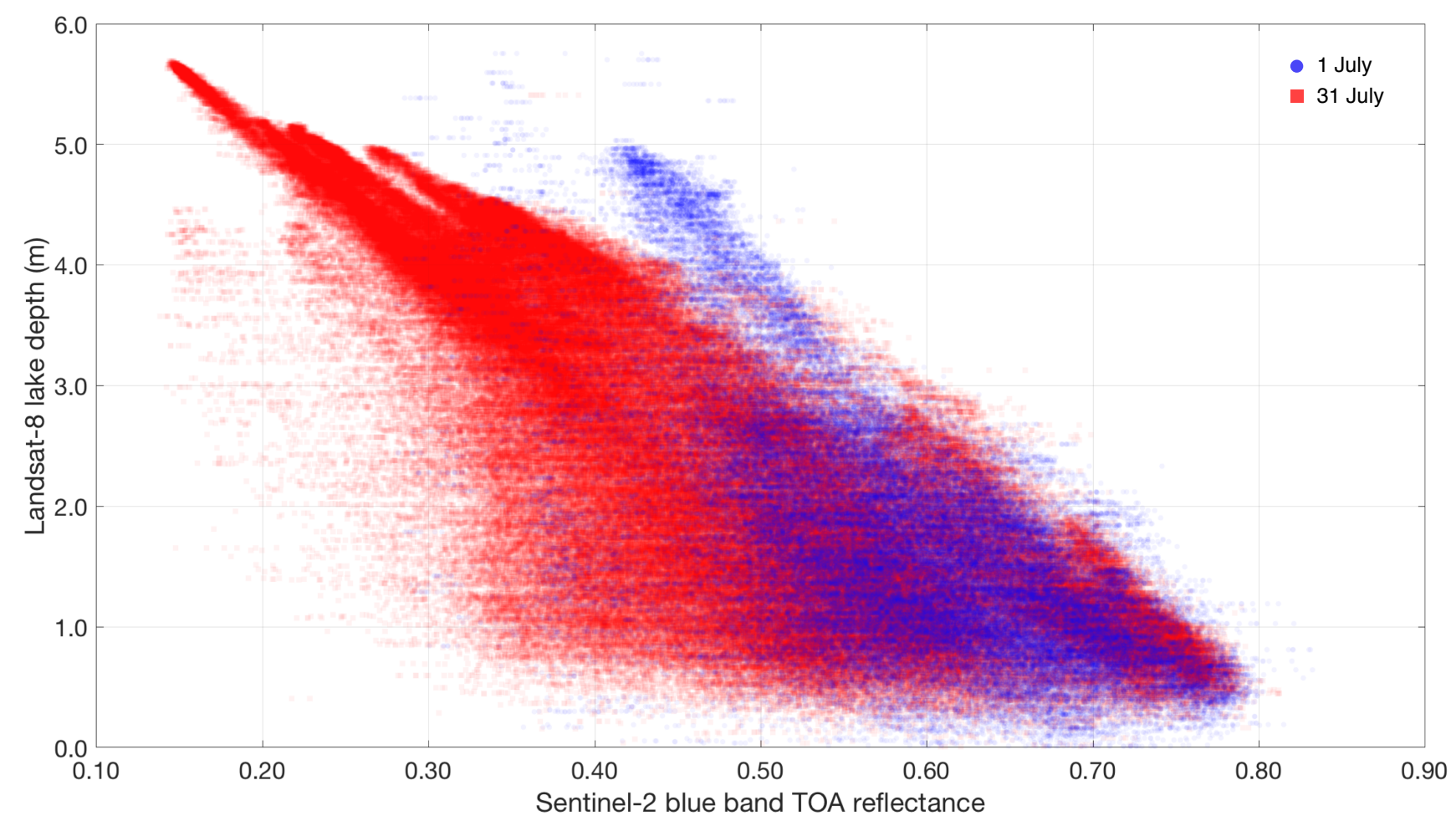

Figure S4: Scatter plot to show the relationship between Sentinel-2 blue band TOA reflectance and Landsat 8 lake depth for 430,650 data points.

\section{References}

Barsi, J. A., Lee, K., Kvaran, G., Markham, B. L., and Pedelty, J. A.: The spectral response of the Landsat-8 Operational Land Imager, Remote Sens., 6, 10232-10251, https://doi.org/10.3390/rs61010232, 2014.

ESA: Sentinel-2 spectral response functions (S2-SRF), accessed 19 February 2018, https://earth.esa.int/web/sentinel/userguides/sentinel-2-msi/document-library/-/asset_publisher/Wk0TKajiISaR/content/sentinel-2a-spectral-responses, 2017.

Maritorena, S., Morel, A., and Gentili, B.: Diffuse reflectance of oceanic shallow waters: Influence of water depth and bottom albedo, Limnol. Oceanogr., 39, 1689-1703, https://doi.org/10.4319/1o.1994.39.7.1689, 1994.

Mobley, C. D.: Light and water: Radiative transfer in natural waters, Academic Press, San Diego, 1994.

Pope, A., Scambos, T. A., Moussavi, M., Tedesco, M., Willis, M., Shean, D., and Grigsby, S.: Estimating supraglacial lake depth in West Greenland using Landsat 8 and comparison with other multispectral sensors, The Cryosphere, 10, 15-27, https://doi.org/10.5194/tc-10-15-2016, 2016.

Pope, R. M. and Fry, E. S.: Absorption spectrum (380-700 nm) of pure water. II. Integrating cavity measurements, Appl. Opt., 36, 8710-8723, https://doi.org/10.1364/AO.36.008710, 1997.

Smith, R. C. and Baker, K. S.: Optical properties of the clearest natural waters (200-800 nm), Appl. Opt., 20, 177-184, https://doi.org/10.1364/AO.20.000177, 1981.

Sneed, W. A. and Hamilton, G. S.: Evolution of melt pond volume on the surface of the Greenland Ice Sheet, Geophys. Res. Lett., 34, L03501, https://doi.org/10.1029/2006GL028697, 2007. 